Portland State University

PDXScholar

$12-1-2010$

\title{
Apertureless near-field/far-field CW two-photon microscope for biological and material imaging and spectroscopic applications
}

\author{
Derek Brant Nowak \\ Portland State University \\ Andrew James Lawrence \\ Portland State University
}

Erik J. Sánchez

Follow this and additional works at: https://pdxscholar.library.pdx.edu/phy_fac

Part of the Physics Commons

Let us know how access to this document benefits you.

\section{Citation Details}

Nowak, D., Lawrence, A., \& Sánchez, E. (2010). Apertureless near-field/far-field CW two-photon microscope for biological and material imaging and spectroscopic applications. Applied Optics, 49(35), 6766-6771. 


\title{
Apertureless near-field/far-field CW two-photon microscope for biological and material imaging and spectroscopic applications
}

\author{
Derek B. Nowak, A. J. Lawrence, and Erik J. Sánchez* \\ Department of Physics, Portland State University, P.O. Box 751, Portland, Oregon 97207, USA \\ *Corresponding author: esanchez@pdx.edu
}

Received 5 August 2010; revised 1 November 2010; accepted 2 November 2010; posted 3 November 2010 (Doc. ID 132969); published 9 December 2010

\begin{abstract}
We present the development of a versatile spectroscopic imaging tool to allow for imaging with singlemolecule sensitivity and high spatial resolution. The microscope allows for near-field and subdiffractionlimited far-field imaging by integrating a shear-force microscope on top of a custom inverted microscope design. The instrument has the ability to image in ambient conditions with optical resolutions on the order of tens of nanometers in the near field. A single low-cost computer controls the microscope with a field programmable gate array data acquisition card. High spatial resolution imaging is achieved with an inexpensive CW multiphoton excitation source, using an apertureless probe and simplified optical pathways. The high-resolution, combined with high collection efficiency and single-molecule sensitive optical capabilities of the microscope, are demonstrated with a low-cost CW laser source as well as a mode-locked laser source. (C) 2010 Optical Society of America
\end{abstract}

OCIS codes: $\quad 180.4243,180.5810,180.4315,180.2520$.

\section{Introduction}

Binnig and Rohrer started a revolution with their invention of the scanning tunneling microscope [1]. The piezo-based technology blossomed into the atomic force microscope [2], which placed fewer constraints on the sample. Karrai and Grober developed the technique of replacing the laser feedback system with a quartz tuning-fork feedback mechanism as a means of maintaining distance between the sample and tip [3]. Tuning-fork shear-force microscopy (SFM) feedback has become popular in the scientific community due to the high degree of customization and versatility. Tuning-fork oscillators are used in many different microscopes from near-field microscopy to in-situ liquid imaging [4], ultrasonic imaging [5], low-temperature imaging $[\overline{6}]$, and magnetic force imaging $[7,8]$, to name a few. In particular, using SFM with near-field microscopes [9-11] has reduced optical resolutions to tens of nanometers.

0003-6935/10/356766-06\$15.00/0

(C) 2010 Optical Society of America
Traditional light microscopy suffers from a diffraction limit that limits the spatial resolution to $\lambda / 2$. With the addition of confocal or nonlinear excitation techniques, this resolution can be enhanced to $\lambda / 3$. Recent advances in far-field super-resolution methods have allowed for optical images beyond the diffraction limit using photo-activated fluorescence molecules [12-16]. These methods rely on computational numerical techniques or tuned stimulated emission with functionalized molecules. Near-field optical microscopes have extended these resolutions below $20 \mathrm{~nm}[17,18]$. Using a combination of a shearforce scanning probe microscope and an inverted optical microscope, the near-field imaging probes are specially shaped metal tips that are illuminated through the objective lens of the inverted optical microscope. This technique has been named tipenhanced near-field optical microscopy (TENOM) [19].

The principle in TENOM is the use of excitation light in the proper polarization to induce a strong localized enhanced field at the tip. The enhanced field consists mainly of nonpropagating (evanescent) components and is, thus, strongly confined to the end 
of the metal tip. The spectroscopic response mechanism for TENOM imaging is normally dependent on the proper use of an electron plasma-supporting probe (typically metal). For biological imaging, $\mathrm{Au}$ probes are used to minimize chemical reactions with the biological specimen (Ag results in a stronger enhancement but reacts with sulfur compounds). The $\mathrm{Au}$ probes are chemically etched and then tailored to specific geometries with the use of a focused ion beam (FIB) [20]. An FIB system allows for the nanofabrication of materials through milling and chemical deposition at the nanometer scale [21]. Newer FIB systems include both electron and ion columns for more precise work [22]. Furthermore, the tips are illuminated by a two-photon excitation (TPE) source, which improves contrast due to a quadratic emission profile. This experimental arrangement can be used for excitation of molecular fluorescence, surface enhanced Raman scattering [23-25], and other forms of linear [26,27] and nonlinear imaging [28]. The detected near-field signal from TENOM probes is typically orders of magnitude greater than the background excitation light. With this increased field, the time for imaging of the fluorophores with an adequate signal-to-noise ratio is reduced. The ultimate resolution obtainable by TENOM is primarily dependent on the geometry of the tip [29,30].

Traditionally, a mode-locked $\overline{(\mathrm{M}} \overline{\mathrm{L})}$ femtosecond pulsed laser would be used as the TPE source [31]. Hell and colleagues explored the use of a CW laser as a TPE source using fiber-based scanning near-field optical microscopy and far-field techniques [32]. They showed theoretically that an increase in average power at the sample of 2 orders of magnitude with a CW source should generate the same TPE emission levels as the high peak pulse power of the ML Ti:sapphire laser. High collection efficiency and low power requirements for this TENOM imaging system (tens of $\mu \mathrm{Ws}$ ) allow for the use of CW TPE sources as a low-cost alternative to pulsed lasers.

The original motivation for the development of a TENOM-specific microscope was the lack of a commercially available shear-force (tuning-fork feedback) system coupled with TPE. Many systems require integration of multiple hardware and computer systems from different vendors. During development, it was determined that creating an optical system with high photon collection efficiency (simplified optical pathways) and ease of customization was highly desirable.

\section{Integrated SFM and Inverted Microscope Design}

A major complication in the alignment of an epiilluminated apertureless near-field microscope is positioning the probe in the focal spot of the excitation laser. To overcome this complication, the scan head houses a piezo stack comprised of a $z$ piezo tube (Boston Piezo-Optics, PZT-5A) and an $x-y$ outersegmented tube with a solid inner ground layer (Boston Piezo-Optics, PZT-5A segmented). These tubes are epoxied together and held in a flexure mount, with MACOR used as an insulator. The flexure mount (Newport MFM-075) allows for coarse alignment (several millimeters) of the tip into the center of the objective lens when viewed through a Bertrand lens system (Thorlabs AC245-050-A and A375TM-A lenses). The $x-y$ outer-segmented piezo tube then allows for precise nanometer positioning of the tip over tens of micrometers for placement in the diffraction-limited focus of the excitation laser. Maximum field enhancement is obtained by positioning the probe with the $x-y$ piezo for maximum photon emission from the sample. The scan head is mounted to a vertical translation stage (Thorlabs LNR50), the position of which is controlled with a 40 threads per inch lead screw (Universal Thread). The vertical position of the scan head is controlled by a stepper motor (Oriental Motor PK243B1A-SG36) with $0.01^{\circ}$-per-step precision and 36:1 gear reduction. Shear-force imaging and optical data collection are accomplished with a National Instruments LabVIEW, PCI-7833R data acquisition card (DAQ) and analog support electronics.

A modular inverted microscope was built using primarily Thorlabs parts. Some parts were custom designed with cast iron and aluminum while maintaining radial symmetry; this was required due to the thermal expansion tolerances that are required for shear-force microscopy, based on previous scanning tunneling microscope (STM) designs [33-35]. The microscope was initially built in a virtual threedimensional environment using SolidWorks 2009 CAD software to ensure fit and function; see Fig. 1(b). Final placement of optics was determined both virtually and experimentally. The microscope is designed primarily for an infinity-corrected objective lens (Olympus 1.4 NA, 60x) but can easily accommodate other objectives. Transmissive samples are raster scanned above the objective on a closed loop scan bed (Physik Instrumente P-733.2CL). The use of tube lenses (Thorlabs SM1L30) allows for simple adjustment of the lens placement to adapt to other focal length objectives and direct mounting of CCD cameras. Inexpensive CCDs (KT\&C KPC-S500B) are adapted to fit with the tube lenses and are used to align the approach of the probe onto the surface, and ultimately into the diffraction-limited laser spot; see Fig. 2.

Excitation light is expanded to fill the back aperture of the objective lens to achieve a high numerical aperture. A periscope optical configuration is used for alignment into the objective through an excitation filter (to remove unwanted laser lines) and a dichroic filter (Chroma 725dcspxr). There are two collection paths in the microscope. The first path allows $92 \%$ of the light to be passed through filters (Chroma ET750sp-2p8), which is then directly coupled into a fiber optic. This allows for direct fiber coupling to a single-photon avalanche photodiode (SPAPD) (Perkin Elmer SPCM-AQR-14-FC) or monochromator (Acton SP-150) with attached intensified CCD (Princeton Instruments Pentamax-512- 


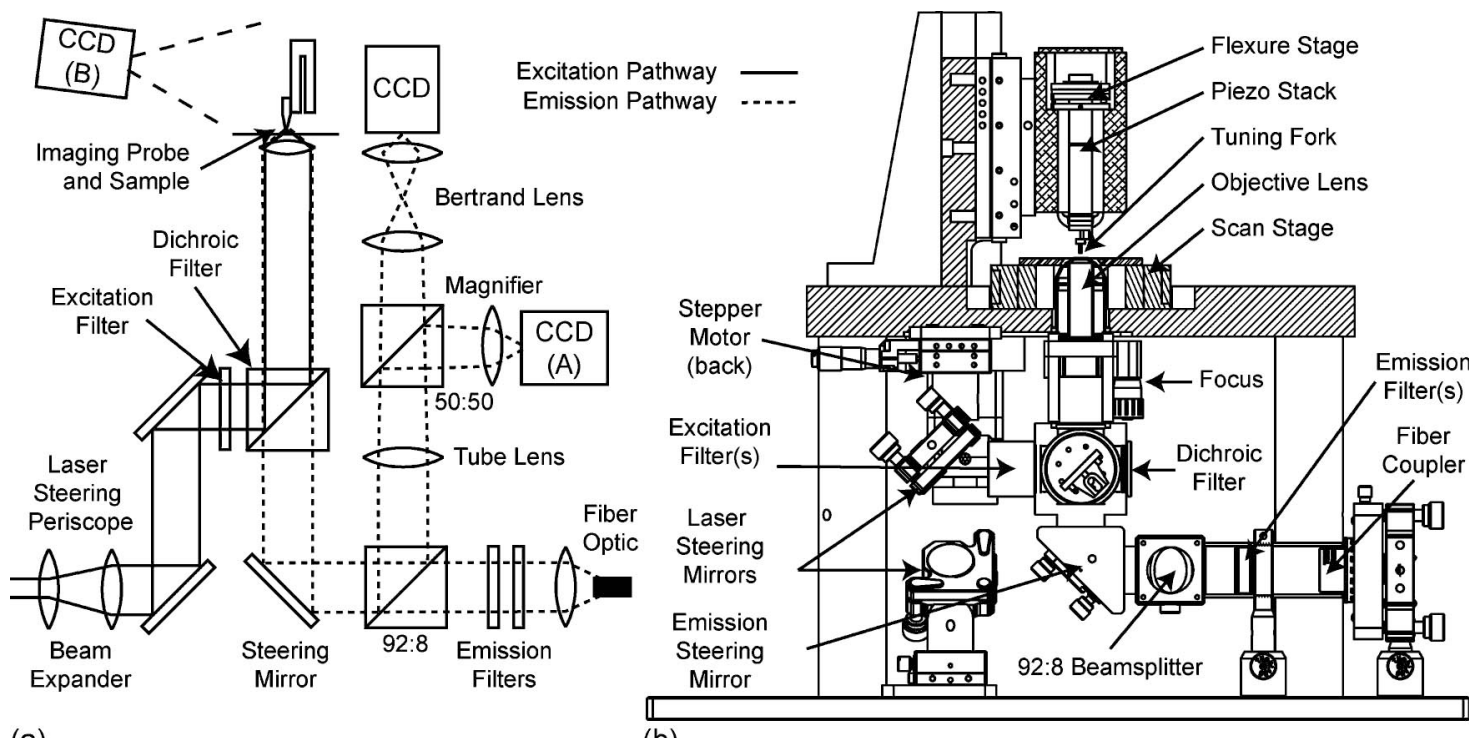

(a)

(b)

Fig. 1. (a) Diagram of the optical pathways of the TENOM system. The beam expander and laser steering periscope give the degrees of freedom necessary to properly fill the back aperture of the objective lens. (b) Mechanical drawing of a cross section of the custom inverted microscope and SFM scan head.

EFT/1EIA). The remaining $8 \%$ is focused with a $200 \mathrm{~mm}$ lens (Thorlabs AC254-200). This signal is split evenly between two of the CCDs-one for imaging the sample surface and one that is equipped with a Bertrand lens system for a fish-eye view of the imaging probe above the surface for tip focus alignment; see Figs. 1 and 2 . The quantum efficiency of the microscope can be calculated by determining all of the optical losses in the system. Using $532 \mathrm{~nm}$ light, the following transmission efficiencies were determined experimentally: objective lens throughput $(78 \%)$, emission collection efficiency $(4 \pi)(38 \%)$, dichroic $(94 \%)$, steering mirror (98\%) emission filters

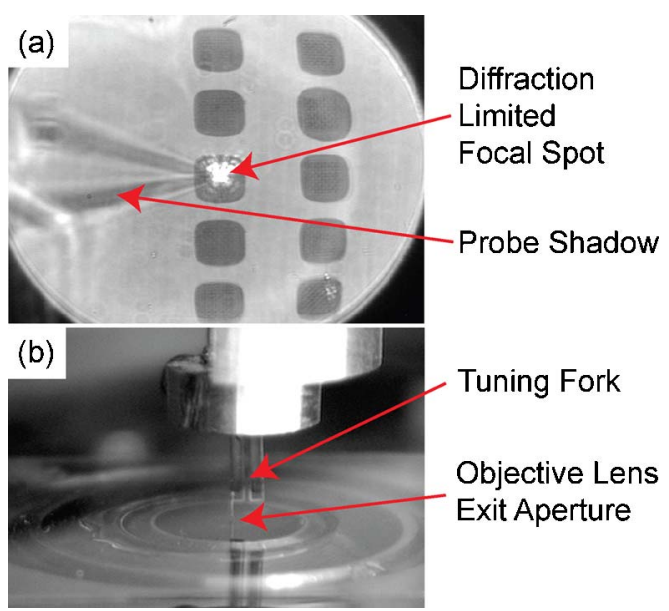

Fig. 2. (Color online) Views from the alignment CCDs: (a) is from the CCD focused on the sample surface with probe side illuminated with LEDs. (The squares are gold coatings made through lithography techniques.); (b) is from the CCD mounted to the scan head or microscope base plate, focusing on the tuning fork and imaging probe. [(a) and (b) correspond to the labels (A) and (B), respectively, in Fig. 1.]
( $3 \times 96 \%)$, beam splitter (90\%), fiber optic coupling and fiber $(85 \%)$, and SPAPD $(55 \%$ at $633 \mathrm{~nm})$. These all contribute to a cumulative throughput of $\sim 10.2 \%$.

\section{Far-Field TPE Imaging}

The sensitivity of the microscope allows for the direct imaging of single molecules at room temperature and atmospheric pressure; see Fig. 3. Imaging at this level has been demonstrated with an ML Ti:sapphire laser [36] (Del Mar Photonics Trestles 50). Both ML pulsed mode and CW mode imaging of single molecules are shown. The single-molecule sample was created with Rhodamine 6G molecules diluted in methanol to $0.1 \mathrm{nM}$ concentration and spin coated at $4000 \mathrm{rpm}$. The Si quantum dots (Nanosys) were spin coated at $4000 \mathrm{rpm}$.

Figure 4 shows a multiphoton image of bovine pulmonary artery endothelial (BPAE) cells excited with an ML Ti:sapphire laser while using the installed spectrograph. All fluorescence from both MitoTracker red CMXRos and Alexa Fluor 488 (Phalloidin) were collected by the SPAPD detector. In Fig. $\underline{4}$, MitoTracker Red stains the F-actin (green fluorescence emission) and Alexa Fluor 488 stains the nuclear DNA. Figure 5 demonstrates the quadratic emission profile for two-photon excitation, using $\mathrm{J}$ aggregates of polyvinyl sulfate (PVS) and pseudoisocyanine (PIC) dye (further discussion in Section 4).

\section{Near-Field TPE CW Imaging}

A sample that lends itself well to successful excitation by the multiphoton process is aggregates of a polymer (PVS) with PIC dye [37,38]. The spectrum (Fig. 6) shows the expected emission for the J aggregates with the two-photon nonlinear excitation process. Using CW illumination requires, on average, about $100 \times$ the power that is used in ML illumina- 


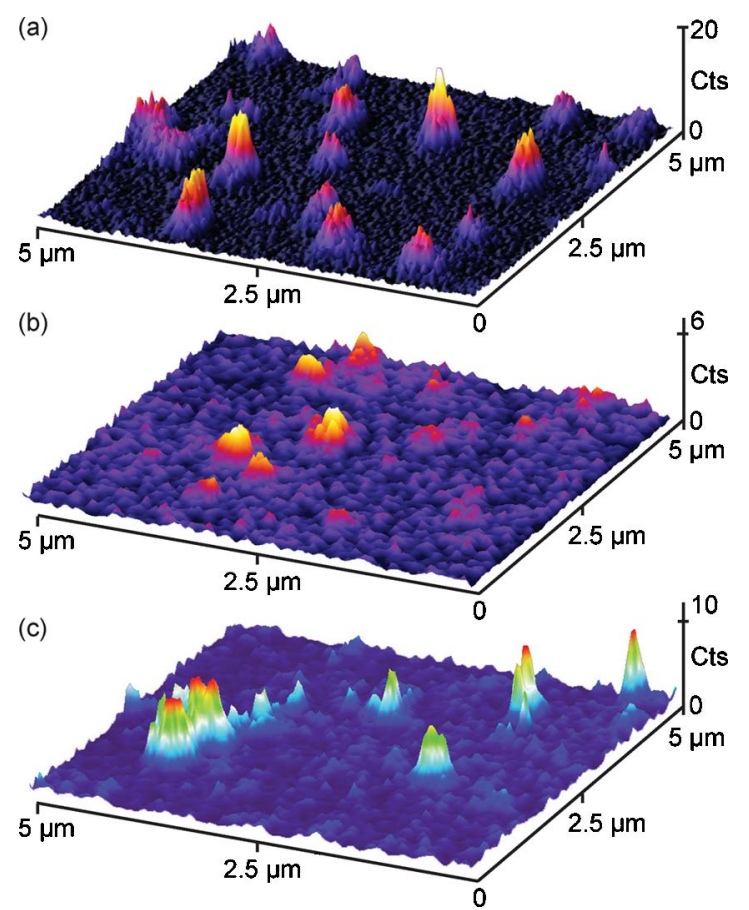

Fig. 3. (Color online) Single-molecule sensitivity: Images (a) and (b) are far-field optical responses of two-photon excitation of single Rhodamine 6G molecules. (a) was acquired with $50 \mathrm{fs}$ laser pulses at $824 \mathrm{~nm}$ and an excitation power of $P_{\text {avg }}=563 \mu \mathrm{W}$. The FWHM of the single molecules is $275 \mathrm{~nm}$, roughly $\lambda / 3$, which is expected for two-photon excitation. (b) was acquired with the same laser configured for $\mathrm{CW}$ at $817 \mathrm{~nm}$ at $P_{\mathrm{avg}}=32 \mathrm{~mW}$. (c) shows twophoton excited Si quantum dots.

tion. To show that the increase in power needed would not cause damage to either the sample or the imaging probe, a region of interest of $\mathrm{J}$ aggregates was imaged with an $\mathrm{ML}$ source at $P_{\text {avg }}=$ $10 \mu \mathrm{m}$ and again with a CW source (SDL-5411-G1) at $P_{\text {avg }}=1.3 \mathrm{~mW}$, while using the same imaging probe. The wavelength was held constant at $833 \mathrm{~nm}$. The acquisition time per $512 \times 512$ image is on the order of $25 \mathrm{~min}$ with a scan rate of $0.35 \mathrm{~Hz}$. The main difference in the imaging between the CW and ML is the increased far-field contribution from the CW illumination. Figure $\underline{6}$ shows these differences in nearfield imaging between ML and CW lasers. This could be improved by the addition of optics to further correct the beam profile of the $\mathrm{CW}$ diode laser.

The TENOM imaging probe was custom built using Au wire with $100 \mu \mathrm{m}$ thickness (Sigma-Aldrich \#349283). The Au wire is chemically etched to an end radius of $\sim 500 \mathrm{~nm}$ and then mounted to the quartz tuning fork (Fox Electronics NC38LF-327). Further modifications to the probe were preformed in a DB-237 dual beam (scanning electron/FIB) microscope produced by the FEI Company, shown in inlay (iv) of Fig. 6. The design of the probe aids in the delocalization of the incident field to the small protrusion on the cone. This design requires that the sample of interest have minimal topography. Improvements in this tip design to yield higher field
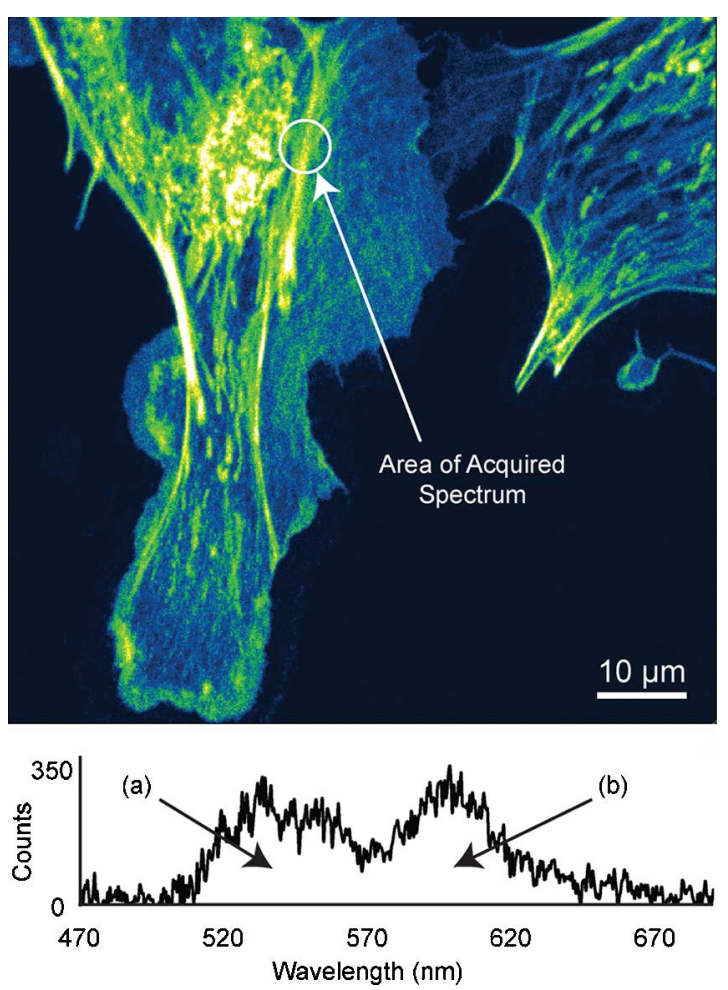

Fig. 4. (Color online) Two-photon far-field fluorescence mapping of BPAE cells mounted on a cover glass slip. Sample was excited at $800 \mathrm{~nm}$ using $50 \mathrm{fs}$ laser pulses with an average intensity of $P_{\text {avg }}=112 \mu \mathrm{W}$. Image shows combined fluorescence of MitoTracker red CMXRos, Alexa Fluor 488 (Phalloidin) and di(2-ethylhexyl) phthalate labels. The spectrum of (a) MitoTracker red CMXRos and (b) Alexa Fluor 488 (Phalloidin) were acquired with a $1.0 \mathrm{~s}$ integration time at the area marked in the image.

enhancements to minimize the far-field contribution during imaging and improve surface topography measurements are currently being investigated.

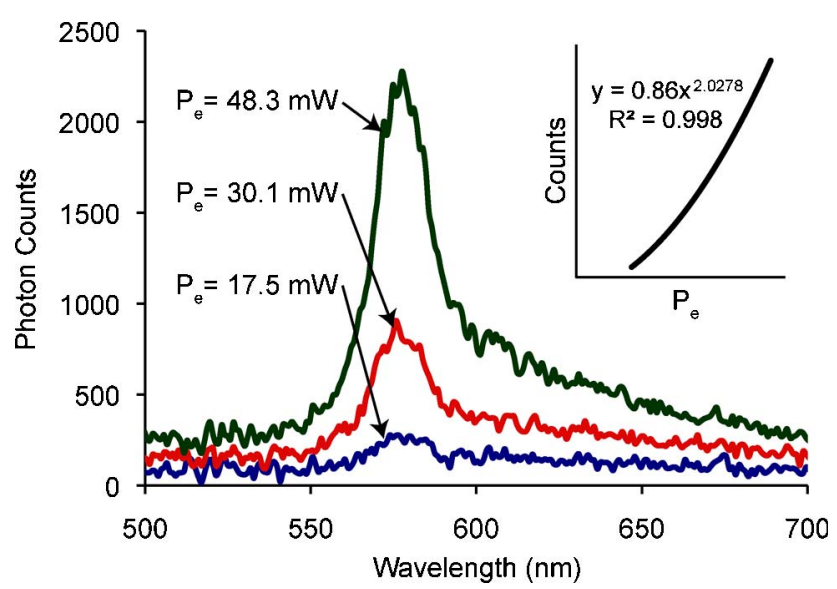

Fig. 5. (Color online) Two-photon CW excited spectra of J aggregates demonstrating the quadratic emission profile of the spectra. Data were obtained using $833 \mathrm{~nm}$ excitation wavelength with an ICCD using a $1.0 \mathrm{~s}$ integration time. The emission output was a power factor of 2.028 in relation to the average excitation power shown by the inlayed graph. 


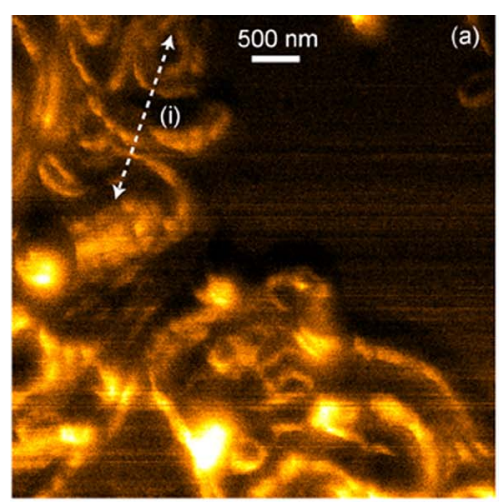

Cts/pixel 0

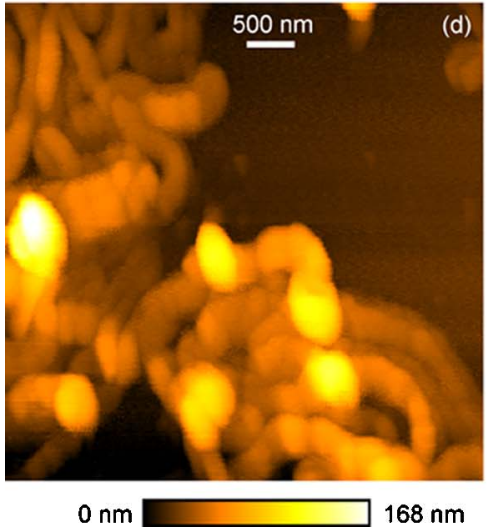

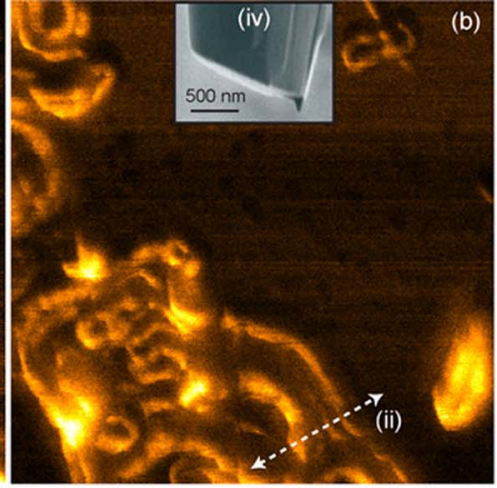

Cts/pixel 0
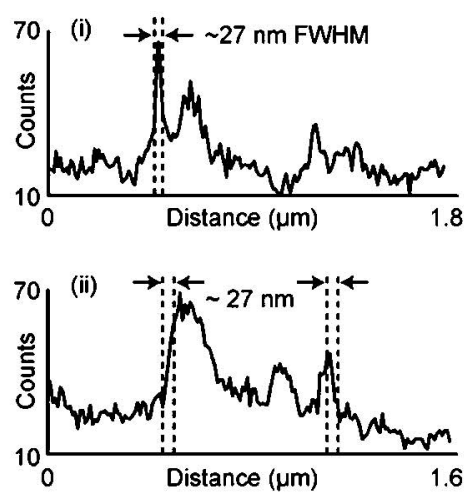

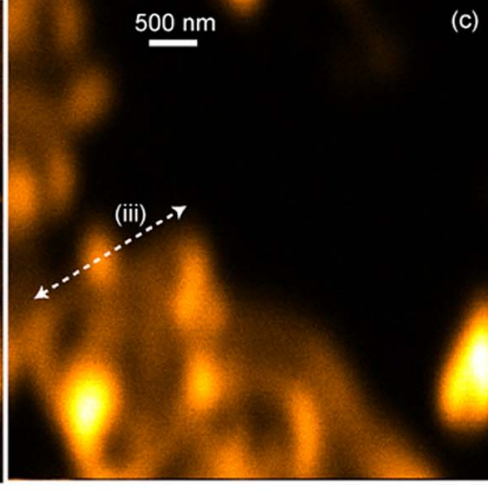

Cts/pixel 0
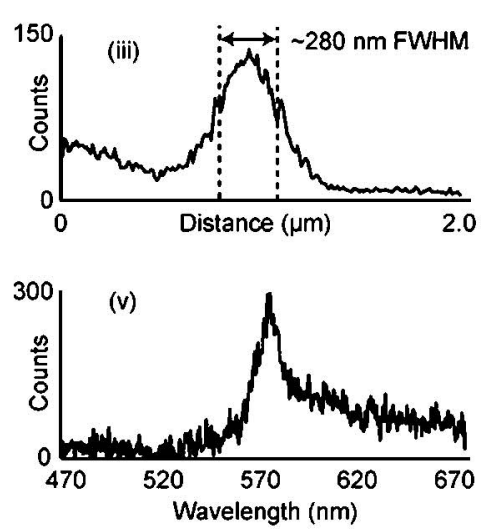

Fig. 6. (Color online) Comparison between near-field imaging with ML and CW excitation. (a) Near-field CW two-photon excitation fluorescence image of J aggregates of PIC dye in a PVS film on a glass substrate. $\lambda_{e}=834 \mathrm{~nm}$ at $P_{\text {avg }}=1.3 \mathrm{~mW}$. (b) Same region of interest (ROI) with $\lambda_{e}=833 \mathrm{~nm}$ at $P_{\mathrm{avg}}=10.2 \mu \mathrm{m}$ ML at $52 \mathrm{fs}$. (c) Diffraction-limited far-field two-photon excitation fluorescence of same ROI and excitation source as (a) at $P_{\text {avg }}=31.5 \mu \mathrm{W}$. (d) Topography of J aggregates taken simultaneously with optical image. Inlay (iv) is an FIB image of the imaging probe. Spectrum (v) is the fluorescence emission from the PIC dye.

\section{Conclusion}

The TENOM system has shown single-molecule sensitivity and near-field imaging at resolutions an order of magnitude beyond the diffraction limit. By developing the system in a standard programming environment with commercially supported, off-theshelf components, combined with simplified usability, we hope to spark interest in the scientific community with this microscope design. The system design could easily accommodate other imaging modes, such as the incorporation of Raman, Kerr magneto-optical imaging capabilities, and other nonlinear imaging techniques.

The addition of modified scanning algorithms would easily allow for other functionalities, such as electric and magnetic shear-force imaging as well. Upon completion of the documentation of this project, the authors intend to release all relevant documentation and software in an open system environment $[\underline{39}, \underline{40}]$.

The authors gratefully acknowledge the support of the National Science Foundation (NSF) through award DBI-0500812 for Biological Sciences Instrument Development. Tip fabrication was assisted through NSF support (ECCS-NSF-0520891), with additional support from PSU and ONAMI, and a
NSF DMR REU site grant (NSF-069280). We are grateful to Matt Spiegelberg from National Instruments for his field-programmable gate array expertise and Analog Devices for help with integrated circuit designs. The authors also thank Marc Nisenfeld and Chuck Heino of Portland State University for machining assistance and Aurelie Snyder from the MMI Core Image Facility at OHSU for the BPAE sample. Financial assistance to finalize the microscope scanning algorithms and electronic design was provided by the Western Institute for Nanoelectronics (WIN) and the Nanoelectronics Research Initiative (NRI).

\section{References}

1. G. Binnig, H. Rohrer, Ch. Gerber, and E. Weibel, "Surface studies by scanning tunneling microscopy," Phys. Rev. Lett. 49, 57-61 (1982).

2. G. Binnig, C. F. Quate, and Ch. Gerber, "Atomic force microscope," Phys. Rev. Lett. 56, 930-933 (1986).

3. K. Karrai and R. D. Grober, "Piezo-electric tuning fork tipsample distance control for near field optical microscopes," Appl. Phys. Lett. 66, 1842-1844 (1995).

4. W. H. J. Rensen, N. F. van Hulst, and S. B. Kammer, "Imaging soft samples in liquid with tuning fork based shear force microscopy," Appl. Phys. Lett. 77, 1557-1559 (2000).

5. A. La Rosa, X. Cui, J. McCollum, N. Li, and R. Nordstrom, "The ultrasonic/shear-force microscope: integrating ultrasonic 
sensing into a near-field scanning optical microscope," Rev. Sci. Instrum. 76, 093707 (2005).

6. J. Rychen, T. Ihn, P. Studerus, A. Herrmann, K. Ensslin, H. J. Hug, P. J. A. van Schendel, and H. J. Gäuntherodt, "Forcedistance studies with piezoelectric tuning forks below 4.2 K," Appl. Surf. Sci. 157, 290-294 (2000).

7. H. Edwards, L. Taylor, W. Duncan, and A. J. Melmed, "Fast, high-resolution atomic force microscopy using a quartz tuning fork as actuator and sensor," J. Appl. Phys. 82, 980-984 (1997).

8. M. Todorovic and S. Schultz, "Magnetic force microscopy using nonoptical piezoelectric quartz tuning fork detection design with applications to magnetic recording studies," J. Appl. Phys. 83, 6229-6231 (1998).

9. D. W. Pohl, W. Denk, and M. Lanz, "Optical stethoscopy: image recording with resolution $\lambda / 20$,” Appl. Phys. Lett. 44, 651-653 (1984).

10. A. Lewis, M. Isaacson, A. Harootunian, and A. Murry, "Development of a $500 \AA$ spatial resolution light microscope: I. Light is efficiently transmitted through $\lambda / 16$ diameter apertures," Ultramicroscopy 13, 227-231 (1984).

11. E. Betzig, J. K. Trautman, T. D. Harris, J. S. Weiner, and R. L. Kostelak, "Breaking the diffraction barrier: optical microscopy on a nanometric scale," Science 251, 1468-1470 (1991).

12. M. Dyba and S. Hell, "Focal spots of size $\lambda / 23$ open up far-field florescence microscopy at $33 \mathrm{~nm}$ axial resolution," Phys. Rev. Lett. 88, 163901 (2002).

13. K. I. Willig, S. O. Rizzoli, V. Westphal, R. Jahn, and S. W. Hell, "STED microscopy reveals that synaptotagmin remains clustered after synaptic vesicle exocytosis," Nature 440, 935-939 (2006).

14. M. J. Rust, M. Bates, and X. Zhuang, "Sub-diffraction-limit imaging by stochastic optical reconstruction microscopy (STORM)," Nat. Methods 3, 793-795 (2006).

15. E. Betzig, G. H. Patterson, R. Sougrat, O. W. Lindwasser, S. Elenych, J. S. Bonifacino, M. W. Davidson, J. LippincottSchwartz, and H. F. Hess, "Imaging intracellular fluorescent proteins at nanometer resolution," Science 313, 1642-1645 (2006).

16. S. T. Hess, T. P. K. Girirajan, and M. D. Mason, "Ultra-high resolution imaging by fluorescence photoactivation localization microscopy," Biophys. J. 91, 4258-4272 (2006).

17. Y. Inouye and S. Kawata, "Near-field scanning optical microscope with a metallic probe tip," Opt. Lett. 19, 159-161 (1994).

18. F. Zenhausern, Y. Martin, and H. K. Wickramsinghe, "Scanning interferometric apertureless microscopy: optical imaging at 10 angstrom resolution," Science 269, 1083-1085 (1995).

19. E. J. Sánchez, L. Novotny, and X. S. Xie, "Near-field fluorescence microscopy based on two-photon excitation with metal tips," Phys. Rev. Lett. 82, 4014-4017 (1999).

20. M. J. Vasile, D. A. Grigg, J. E. Griffith, E. A. Fitzgerald, and P. E. Russell, "Scanning probe tips formed by focused ion beams," Rev. Sci. Instrum. 62, 2167-2171 (1991).

21. J. Orloff, "High-resolution focused ion beams," Rev. Sci. Instrum. 64, 1105-1130 (1993).

22. E. J. Sánchez, J. T. Krug II, and X. S. Xie, "Ion and electron beam assisted growth of nanometric SimOn structures for near-field microscopy," Rev. Sci. Instrum. 73, 3901-3907 (2002).

23. J. Wessel, "Surface-enhanced optical microscopy," J. Opt. Soc. Am. B 2, 1538-1541 (1985).

24. R. M. Stöckle, Y. D. Suh, V. Deckert, and R. Zenobi, "Nanoscale chemical analysis by tip-enhanced Raman spectroscopy," Chem. Phys. Lett. 318, 131-136 (2000).

25. A. Hartschuh, E. J. Sánchez, X. S. Xie, and L. Novotny, "High-resolution near-field Raman microscopy of singlewalled carbon nanotubes," Phys. Rev. Lett. 90, 095503 (2003).

26. J. M. Gerton, L. A. Wade, G. A. Lessard, Z. Ma, and S. R. Quake, "Tip-enhanced fluorescence microscopy at 10 nanometer resolution," Phys. Rev. Lett. 93, 180801 (2004).

27. H. F. Hamann, A. Gallagher, and D. J. Nesbitt, "Near-field fluorescence imaging by localized field enhancement near a sharp probe tip," Appl. Phys. Lett. 76, 1953-1955 (2000).

28. T. Ichimura, N. Hayazawa, M. Hashimoto, Y. Inouye, and S. Kawata, "Tip-enhanced coherent anti-Stokes Raman scattering for vibrational nanoimaging," Phys. Rev. Lett. 92, 220801 (2004).

29. J. T. Krug, E. J. Sánchez, and X. S. Xie, "Design of near-field optical probes with optimal field enhancement by finite difference time domain electromagnetic simulation," J. Chem. Phys. 116, 10895-10901 (2002).

30. L. Novotny, E. J. Sánchez, and X. S. Xie, "Near-field optical imaging using metal tips illuminated by higher-order HermiteGaussian beams," Ultramicroscopy 71, 21-29 (1998).

31. W. Denk, J. H. Strickler, and W. W. Webb, "Two-photon laser scanning fluorescence microscopy," Science 248, 73-76 (1990).

32. S. W. Hell, M. Booth, S. Wilms, C. M. Schettner, A. K. Kirsch, D. J. Arndt-Jovin, and T. M. Jovin, "Two-photon near- and far-field fluorescence microscopy with continuous-wave excitation," Opt. Lett. 23, 1238-1240 (1998).

33. B. Drake, R. Sonnenfeld, J. Schneir, P. K. Hansma, G. Slough, and R. V. Coleman, "A tunneling microscope for operation in air or fluids," Rev. Sci. Instrum. 57, 441-445 (1986).

34. S. Gregory and C. T. Rogers, "High-speed scanning tunneling microscopes," J. Vac. Sci. Technol. A 6, 390-392 (1988).

35. S. Park and R. C. Barrett, "Design considerations for an STM system," in Methods of Experimental Physics, J. A. Stroscio and W. J. Kaiser, eds. (Academic, 1993), Vol. 27, pp. 30-76.

36. E. J. Sánchez, L. Novotny, G. R. Holtom, and S. Xie, "Roomtemperature fluorescence imaging and spectroscopy of single molecules by two-photon excitation," J. Phys. Chem. A 101, 7019-7023 (1997).

37. D. A. Higgins and P. F. Barbara, "Excitonic transitions in Jaggregates probed by near-field scanning optical microscopy," J. Phys. Chem. 99, 3-7 (1995).

38. D. A. Higgins, J. Kerimo, D. A. Vanden Bout, and P. F. Barbara, "A molecular yarn: near-field optical studies of self-assembled, flexible, fluorescent fibers," J. Am. Chem. Soc. 118, 4049-4058 (1996).

39. D. B. Nowak, "ANSOM Project," http://ansom.research.pdx. edu/.

40. D. B. Nowak, "The design of a novel tip enhanced near-field scanning probe microscope for ultra-high resolution optical imaging," Ph.D dissertation (Portland State University, 2010). 